\title{
Primary Spinal Cord Astrocytoma Presenting as Intracranial Hypertension: A Case Report
}

\author{
Sungryong Lim, Seung Joo Lee, Seung Chul Rhim \\ Department of Neurological Surgery, Asan Medical Center, University of Ulsan College of Medicine, Seoul, Korea
}

Increased intracranial pressure (IICP) is rarely seen in association with primary spinal tumors. We describe a 58-year-old man who was diagnosed with a primary spinal cord astrocytoma, who first presented with hypesthesia, followed by intracranial hypertension, papilledema and blurred vision. On first admission, he presented with hypesthesia but without paraparesis, headache or blurred vision. Spinal MRI showed a relatively well-enhanced solid mass with a cystic portion at the cervico-thoracic level, shown histologically to be a grade I pilocytic astrocytoma. After gross total resection of the tumor, the patient had no significant neurological changes. Nine months later, the patient was admitted with headache, blurred vision and paraparesis. An ophthalmologic examination showed papilledema and lumbar tapping revealed IICP. A spinal MRI showed recurrence of the tumor which was found to be a glioblastoma after reexplorative debulking surgery. After resection, his headaches and blurred vision improved, but his paraparesis did not. These findings show that a primary spinal cord astrocytoma may cause IICP.

Key Words: Spinal cord neoplasms · Astrocytoma $\cdot$ Papilledema $\cdot$ Intracranial hypertension

\section{INTRODUCTION}

Primary spinal tumor is uncommon. Patients may present clinically with hypesthesia, paraparesis, voiding difficulties and/ or abnormal reflexes depending on the location of the tumor. Increased intracranial pressure (IICP) associated with primary spinal tumor, however, has rarely been reported. This case report describes the clinical experience of a patient who presented with IICP and rapid pathologic progression due to a primary spinal cord astrocytomas at the cervico-thoracic junction.

\section{CASE REPORT}

In May 2009, a 58 year-old man presented with hypesthesia below the nipple and ataxia without initial paraparesis. Neurologic examination showed decreasing proprioception and pathologic reflexes, such as the Babinski sign. Spinal MRI

- Received: April 30, 2012 - Revised: September 18, 2012

- Accepted: September 25, 2012

Corresponding Author: Seung Chul Rhim, MD, PhD

Department of Neurological Surgery, Asan Medical Center, 388-1,

Poongnap-dong, Songpa-gu, Seoul 138-736, Korea

Tel: +82-2-3010-3555, Fax: +82-2-476-6738

Email: sclim@amc.seoul.kr showed a relatively well enhanced solid mass including a cystic portion at levels T1 to T4 and diffuse spinal cord edema, from the lower medulla to the T11 level (Fig. 1). Myelotomy was performed immediately. The patient's spinal cord was extremely swollen and demarcation between the tumor and the cord was poor (Fig. 2). After gross total resection of the tumor, the patient had no significant neurological changes. Histological examination of a tissue sample revealed a grade I pilocytic astrocytoma, according to the $\mathrm{WHO}$ classification.
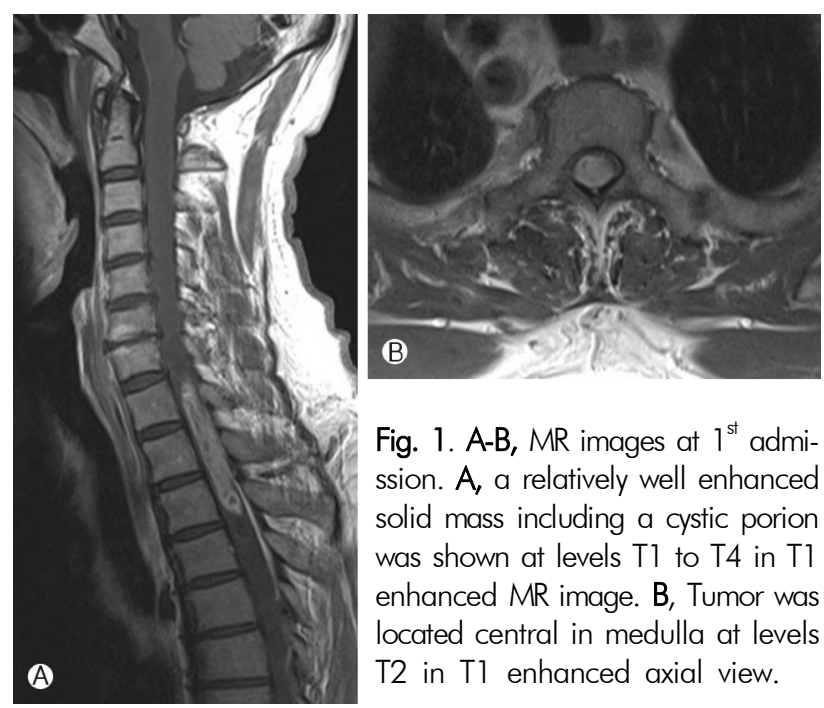

Fig. 1. A-B, MR images at $1^{\text {st }}$ admission. $A$, a relatively well enhanced solid mass including a cystic porion was shown at levels $\mathrm{Tl}$ to $\mathrm{T} 4$ in $\mathrm{Tl}$ enhanced MR image. B, Tumor was located central in medulla at levels $\mathrm{T} 2$ in $\mathrm{T} 1$ enhanced axial view. 


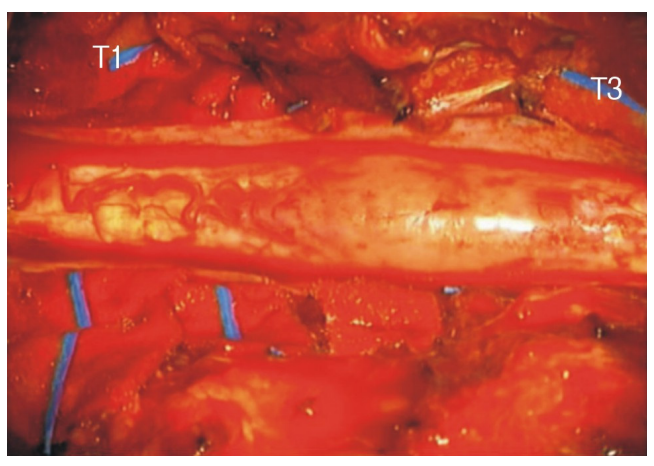

Fig. 2. Spinal cord was swollen severely and demarcation between tumor and cord was poor.
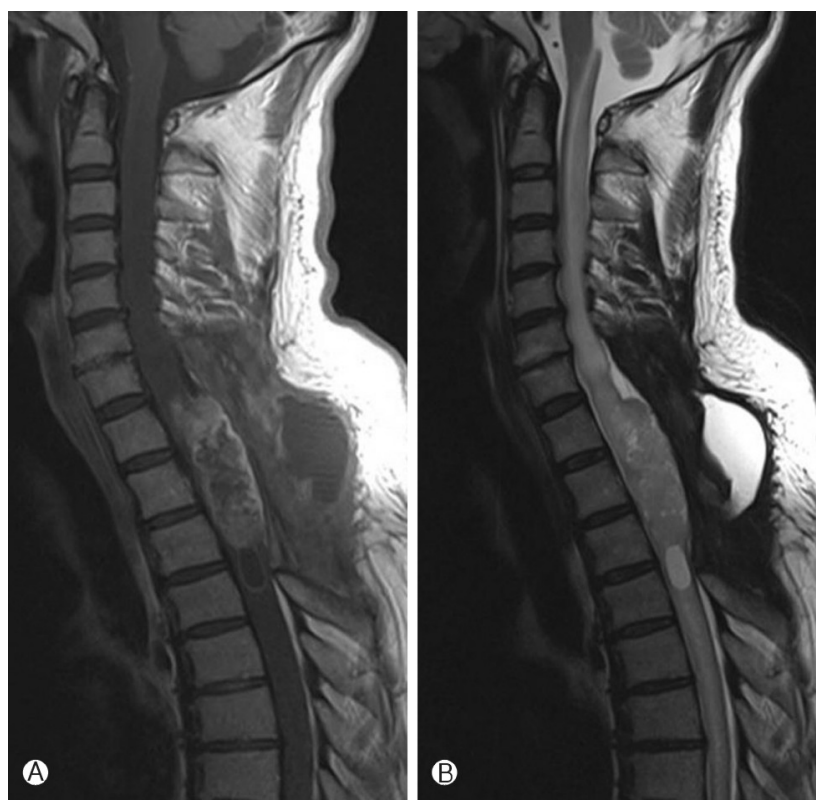

Fig. 3. Postoperative sagittal MR images. (A) enhancing solid and cystic mass in the T1-T3 was increased in T1 enhnaced sagittal MR. (B) high signal intensity was seen from $\mathrm{Cl}$ to $\mathrm{T} 10$ in $\mathrm{T} 2$ sagittal MR image.

In February, 2010, 9 months after the resection, the patient was admitted with headache, blurred vision and paraparesis (Gr II). Brain MRI showed no evidence of abnormal enhancement in brain MRI, but spinal MRI showed a newly developed enhancing lesion involving T1-T3, combined with diffuse spinal cord edema involving C7-T4 and complicated fluid collection at the dorsal aspect of the operation site with no evidence of CSF leakage (Fig. 3). Opthalmologic examination showed papilledema and lumbar tapping showed elevated opening pressure $\left(43 \mathrm{cmH}_{2} \mathrm{O}\right)$. CSF cytology revealed an elevated CSF protein concentration $(343 \mathrm{mg} / \mathrm{dl})$, but no malignant cells. Reexplorative debulking surgery was performed. The specimen showed areas of necrosis as well as microvascular hyperplasia,
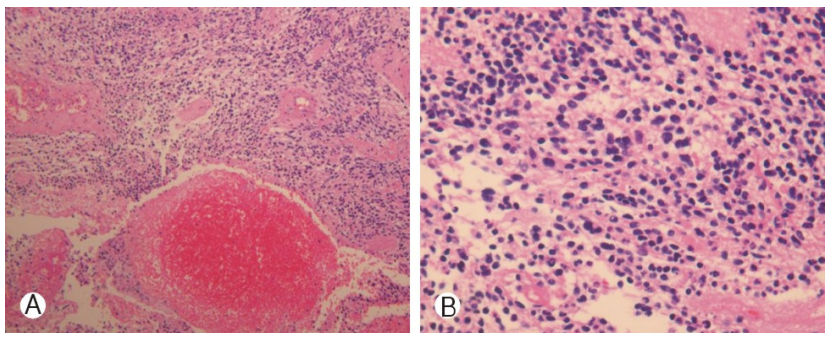

Fig. 4. A-B, Present resection specimen shows area of necrosis as well as microvascular hyperplasia. The cellularity is also higher than pre viously resected specimen. Presence of necrosis and microvascular hyperplasia are features supporting glioblastoma (A, H\&E $40 \times$. B, H\&E 400×).

along with cellularity higher in this than in the previously resected specimen (Fig. 4). Areas of necrosis and microvascular hyperplasia supported a diagnosis of a malignantly transformed glioblastoma. Within a few days of surgery, the patient's headaches and blurred vision improved, but paraparesis persisted. The patient was treated with adjuvant radiation therapy, consisting of a dose of $40 \mathrm{~Gy}$ in 16 fractions.

\section{DISCUSSION}

Increased intracranial pressure rarely occurs in patients with spinal tumors. The causative relationship between spinal lesions and IICP is not completely clear, although any mechanisms have been suggested, including tumor blockage of the CSF pathway, compromised spinal elastic reservoir of CSF, high CSF protein concentration, arachnoiditis and compression of the spinal venous plexus".

Cervico-medullary extension of a rostral tumor cyst may cause obliteration of the subarachnoid pathways at the level of the fourth ventricle outlets ${ }^{10)}$. We performed neither CineMR nor ventriculography for identifying evidence of CSF pathway obstruction, in aggrement with findings in other patients, showing no obstruction of the CSF pathways at the level of the cervico-medullary junction ${ }^{2,3,5)}$. It is very unlikely that the tumor itself directly blocked the absorption of the $\mathrm{CSF}^{9}$. Most CSF is absorbed at the interface between the cortical subarachnoid space and the dural venous sinuses, although several additional sites of CSF absorption have been suggested. Other causes of compression of the thecal sac, such as fracture or epidural tumor, have not been associated with either hydrocephalus or pseudotumor cerebri ${ }^{9)}$.

Increased CSF protein concentration is a very common finding in these patients, as in ours. Increased protein concentration would increase CSF viscosity, increasing its resistance to resorption ${ }^{4)}$. Some patients with spinal cord tumors and hydrocephalus or the pseudotumor cerebri syndrome, howe- 
ver, do not have high CSF protein concentrations, and most patients show only modest increases.

Arachnoiditis may explain the association between spinal cord tumors and intracranial hypertension. Several potential chemical markers have been suggested, including fibrinogen, which is converted to fibrin in the CSF. If fibrin creates a blockage at the level of the basal cisterns, it would cause hydrocephalus. If it creates a blockage at the level of the arachnoid villi, it could lead to the pseudotumor cerebri syndrome ${ }^{2)}$. Another candidate biomarker is the inflammatory cytokine transforming growth factor (TGF)-[beta]. Found in a variety of primarily vascular structures such as the choroid plexus, TGF-[beta] is present in high concentrations in platelets and has been shown to induce the proliferation of leptomeningeal cells and scarring at the base of the brain and in the area of the arachnoid villi ${ }^{8}$. Another explanation of arachnoiditis is by intracranial seeding, the theory of "neoplastic arachnoiditis" with associations observed among spinal tumors, intracranial seeding, and hydrocephalus ${ }^{6}$. One study assessed three intraspinal tumors (two malignant schwannomas and one benign oligodendroglioma) associated with hydrocephalus that were attributed to basal cistern adhesions macroscopically evident at autopsy. Microscopic examination showed neoplastic elements in the two patients with schwannomas, suggesting that intracranial spread and proliferation of tumor cells through the subarachnoid pathways may be responsible for hydrocephalus, at least in in some patients ${ }^{2}$. Brain MRI showed no evidence of brain metastases in this patient, and we couldn't identify arachnoiditis without an autopsy. However, this tumor grew aggressively and rapidly became malignant, suggesting that it likely disseminated through the subarachnoid space. This pathophysiological mechanism may explain the association of hydrocephalus with intramedullary high-grade gliomas in patients with intracranial metastases, but the mechanism in patients with low-grade glial tumors remains unclear.

Primary spinal high-grade gliomas are uncommon, with anaplastic astrocytomas accounting for approximately $15 \%$ of all intraspinal tumors, and glioblastoma multiforme (GBM) accounting for only $1-5 \%$ of all $\mathrm{GBM}^{1)}$. In patients with anaplastic astrocytoma who could undergo radical resection, there was a trend toward prolonged survival in the absence of tumor dissemination $^{5}$. In contrast, other researchers suggested that more aggressive resection and radiotherapy of GBM has been associated with poor survival, similar to historical controls of patients who underwent diagnostic biopsy and radiotherapy ${ }^{7}$. We found that radical resection of GBM was helpful in easing our patient's symptoms. Treatment of tumor, however, remains unclear.

\section{CONCLUSION}

Primary spinal astrocytoma is not common and patients may rarely present with IICP symptoms. Many theories were proposed but not explained clearly. Arachnoiditis may explain the cause of IICP symptoms for the primary spinal tumors, especially for the malignant ones. And radical resection was helpful for the IICP symptoms of this patient.

\section{REFERENCES}

1. Andrews AA, Enriques L, Renaudin J, Tomiyasu U: Spinal intramedullary glioblastoma with intracranial seeding. Report of a case. Arch Neurol 35:244-245, 1978

2. Cinalli G, Sainte-Rose C, Lellouch-Tubiana A, Sebag G, Renier D, Pierre-Kahn A: Hydrocephalus associated with intramedullary low-grade glioma. Illustrative cases and review of the literature. J Neurosurg 83:480-485, 1995

3. Eaton LM, Craig WM: Tumor of the spinal cord: Sudden paralysis following lumbar puncture. Proc Staff Meet Mayo Clin 15: 170-172, 1940

4. GARDNER WJ, SPITLER DK, WHITTEN C: Increased intracranial pressure caused by increased protein content in the cerebrospinal fluid; an explanation of papilledema in certain cases of small intracranial and intraspinal tumors, and in the GuillainBarre syndrome. N Engl J Med 250:932-936, 1954

5. Jung SW, Park KH, Kim JO, Kim JC: Cerebellar anaplastic astrocy toma with metastasis to the cauda equina. Korean J Spine 1(2): 263-267, 2004

6. Koshu K, Tominaga T, Fujii Y, Yoshimoto T: Quadraparesis after a shunting procedure in a case of cervical spinal neurinoma associated with hydrocephalus: case report. Neurosurgery 32:669670; discussion 670-1, 1993

7. Maurice-Williams RS, Lucey JJ: Raised intracranial pressure due to spinal tumours: 3 rare cases with a probable common mechanism. Br J Surg 62:92-95, 1975

8. McGirt MJ, Goldstein IM, Chaichana KL, Tobias ME, Kothbauer KF, Jallo GI: Extent of surgical resection of malignant astrocytomas of the spinal cord: outcome analysis of 35 patients. Neurosurgery 63:55-60; discussion 60-61, 2008

9. Motohashi O, Suzuki M, Yanai N, Umezawa K, Shida N, Yoshimoto T: Thrombin and TGF-beta promote human leptomeningeal cell proliferation in vitro. Neurosci Lett 190:105-108, 1995

10. Rekate HL: Why would a spinal tumor cause increased intracranial pressure? J Neuroophthalmol 22:197-198, 2002

11. Rifkinson-Mann S, Wisoff JH, Epstein F: The association of hydrocephalus with intramedullary spinal cord tumors: A series of 25 patients. Neurosurgery 27:749-754; discussion 754, 1990 